\title{
Expectativas de los Doctorandos Chilenos: Insumos para la Elaboración de un Perfil de Egreso Doctoral
}

\author{
Karen P. Núñez-Valdés(1), José A. González ${ }^{(2)}$ y Silvia J. Sarzoza ${ }^{(3)}$ \\ (1) Facultad de Ciencias de la Educación, Programa de Doctorado en Política y Gestión Educativa, Universidad de \\ Playa Ancha, Avenida Playa Ancha \#850, Valparaíso, Chile (e-mail: k.nunez.valdes@gmail.com) \\ (2) Facultad de Ciencias Naturales y Exactas, Departamento de Matemática, Universidad de Playa Ancha, Avenida \\ Playa Ancha \#850, Valparaíso, Chile (e-mail: jgonzalez@upla.cl) \\ (3) Facultad de Ciencias de la Educación, Centro de Estudios Avanzados, Universidad de Playa Ancha, Avenida Playa \\ Ancha \#850, Valparaíso, Chile (e-mail: ssarzoza@upla.cl)
}

Recibido Dic. 12, 2018; Aceptado Feb. 13, 2019; Versión final Mar. 19, 2019, Publicado Oct. 2019

\begin{abstract}
Resumen
En este estudio se propone identificar las expectativas de los doctorandos de las áreas de educación y ciencias sociales chilenos, sobre la formación en este nivel, con la finalidad de obtener insumos para la posterior elaboración de un Perfil de Egreso doctoral que contemple estas expectativas. Para el levantamiento de los datos se confeccionó un instrumento de abstracción mixto que caracteriza tres dimensiones: una dimensión relativa a las expectativas de los doctorandos en términos académicos, otra a las razones de la elección del programa que se cursa y la última a las proyecciones y elementos de contexto como becas y procesos de acreditación. La metodología utilizada es cuantitativa no experimental, de corte transversal sobre las frecuencias. Como resultados, se presentan las expectativas de los doctorandos en cada una de las dimensiones antes señaladas, permitiendo establecer las competencias que ha de contener un programa de doctorado chileno.
\end{abstract}

\section{Expectations of the Chilean Doctoral Students: Inputs for the Preparation of a Doctoral Graduate Profile.}

\begin{abstract}
In this study, it is proposed to identify the expectations of doctoral students in the areas of education and social sciences in Chile, on training at this level, to obtain inputs for the subsequent preparation of a doctoral graduate profile that includes these expectations. To collect the data, a mixed abstraction instrument was developed which characterizes three dimensions: a dimension related to the doctoral students' expectations in academic terms, another to the reasons for the choice of the program being studied and the last one to the projections and context elements such as scholarships and accreditation processes. The methodology used is quantitative, non-experimental, of cross-section over the frequencies. As results, the doctoral students' expectations in each of the aforementioned dimensions are presented, allowing to establish the competences that a Chilean doctorate program must contain.
\end{abstract}

Keywords: expectations; doctorate; graduation profile; accreditation; research 


\section{INTRODUCCIÓN}

En la actualidad, se considera que la educación terciaria es esencial para el fomento del conocimiento y la innovación, ambos necesarios para mantener el crecimiento económico. Varios países miembros de la Organización para la Cooperación y el Desarrollo Económico (OCDE) han puesto énfasis en mejorar la calidad de la educación (Organización para la Cooperación y el Desarrollo Económico, 2017), ya que esta "es un elemento fundamental para modelar la inclusión económica y social en Chile. Una educación de calidad y con acceso equitativo puede contribuir a disminuir los niveles de desigualdad del país" (Ministerio de Educación, 2017). En este contexto, las universidades son cada vez más exigidas, dado el rol que se les ha asignado tanto a nivel económico, como social y cultural. Estas instituciones, a través, del desarrollo del postgrado y en específico de programas doctorales, han intentado responder a las demandas de una sociedad globalizada y compleja (Manzo et al., 2006), mediante la formación de capital humano avanzado "altamente cualificado en investigación y producción científica y tecnológica, representando un valor estratégico de primera magnitud" para el país (Jiménez-Ramírez, 2017). Marvin, citado por Krauskopf (2003) señala que "los doctorados constituyen, ciertamente, el grado superior que confiere una universidad. Los hay académicos y profesionales. El doctorado académico, representado en forma creciente por el Ph. D anglosajón, implica en lo fundamental una investigación rigurosa y profunda y revela que una persona domina los conceptos más avanzados en un área del conocimiento y ha desarrollado la capacidad de contribuir intelectualmente, de modo independiente a este campo del saber". Por su parte, Cardoso, citado por Sánchez-Medina et al., (2016, p. 98), señala que "los estudios de posgrado son la cima de los procesos de formación. Preparan en la metodología y el desarrollo de nuevos conocimientos e innovaciones para la investigación", fundamentales para el siglo XXI, donde "el doctorado es la marca de fábrica, distintiva y exclusiva de la Universidad investigado" (Nebot, 2009, p. 12)

En Chile, la formación doctoral es relativamente reciente, pues durante mucho tiempo las actividades universitarias estuvieron centradas en la formación de licenciados, lo que evidentemente significó una baja tasa de graduación de doctores chilenos en comparación con otros países de la región (Vera, 2010). El primer programa doctoral fue creado por la Universidad de Chile el año 1947, en el área de Filosofía, y con posterioridad a ello se implementaron programas en áreas reconocidas tradicionalmente como científicas, lo que fue seguido por programas de áreas menos desarrolladas, como Ciencias Agrícolas, Ciencias Sociales y Educación (Baeza, 2017). El número de programas doctorales aumentó al igual que todo el sistema universitario chileno, lo que fue posible por el desarrollo de iniciativas estatales tendientes al aumento del número de doctores chilenos, a través, del financiamiento de estudios doctorales tanto en Chile como en el extranjero (Chiappa y Muñoz, 2015). Para Der Weijden et al., (2016) esto obedece a que las universidades ampliaron sus objetivos tradicionales, como lo era el desarrollo del conocimiento y el intercambio, a la comercialización del conocimiento.

Habiendo transcurrido más de siete décadas desde la creación del primer programa de doctorado en Chile, la oferta se ha expandido en áreas, universidades y regiones, lo que ha generado, al igual que en algunos programas de pre-grado (pedagogías y carreras del área de la salud), la necesidad de certificar la calidad de la formación que se está entregando. En la actualidad, la entidad responsable de ello es la Comisión Nacional de Acreditación, conforme a la Ley $\mathrm{N}^{\circ}$ 20.129/2006, que establece el sistema de aseguramiento de la calidad de la Educación Superior en Chile (Cancino y Schmal, 2014). Con la entrada en vigencia de la Ley No 21.091/2018 sobre Educación Superior, se implementarán nuevos mecanismos de evaluación y la calidad de todas las carreras y programas, independiente de las modalidades serán examinada en el proceso de acreditación institucional, dado que se tratará de un proceso integral que aborde la diversidad de la Institución, estableciéndose además que los programas doctorales deberán someterse a procesos de acreditación de forma obligatoria. Cabe señalar, que la acreditación de los doctorados prestigia a las Universidades y permite a los doctorandos acceder a becas mediante la postulación por mérito (Chiappa y Muñoz, 2015), lo que resulta todo un reto para las universidades, ya que el financiamiento es fundamental para orientar la formación de doctorado hacia la investigación (Horgué, 2012), siendo fundamental de cara al futuro poseer la acreditación de los programas doctorales.

Dentro de los criterios por los que son evaluados los programas de Postgrado está el denominado "Características y resultados del Programa", y uno de los elementos constitutivos de este, es el "perfil de egreso", el que debe reflejar con claridad los conocimientos, habilidades y aptitudes que se espera alcancen los graduados con la formación recibida, a partir de las actividades curriculares, la didáctica y la evaluación (Möller y Gómez, 2014). La revisión del "perfil de egreso", debe ser sistemática y formar parte de los mecanismos formales de evaluación del Programa, en ella participan tanto los estudiantes como los académicos y los graduados. Respecto a la elaboración de perfiles, la Comisión Nacional de Acreditación (2013), a través, de la Guía para la Elaboración del Informe de Autoevaluación de los programas de postgrado, señala los aspectos a evaluar, constituyéndose este como un insumo para la elaboración de un perfil de egreso de nivel doctoral. De igual modo, para verificar la pertinencia, la Comisión Nacional de Acreditación 
sugiere utilizar elementos orientadores, referidos a la pertinencia y consistencia entre el nombre del programa y los objetivos de éste, la contribución de las actividades formativas al logro del perfil declarado, entre otros. La evaluación de los criterios para la acreditación se realiza mediante la aplicación de una encuesta de satisfacción respecto a la calidad de la formación, no considerándose las expectativas de los doctorados en formación respecto al grado que aspiran obtener. Tener conocimiento sobre las expectativas de los usuarios de estos programas, puede aportar información sobre los elementos que son más relevantes para la formación doctoral y dar cuenta, eventualmente, de las brechas entre lo que entrega un programa y lo que se espera de él, considerando que hasta mayo de 2018 no existía obligatoriedad de los programas de este nivel a acreditarse y por tanto de rendir cuentas sobre la formación entregada. Corvalán, Falabella y Rojas (2011) en su estudio ya señalaban que existía una desregulación de la educación superior en Chile, lo que acontecía principalmente porque los mecanismos regulativos son insuficientes, especialmente en relación a los estudios de postgrado.

Estudios internacionales como el desarrollado por Shapiro et al., (2017) han indagado sobre la satisfacción del estudiante de doctorado en relación al profesorado y a los temas de investigación. Sus resultados muestran que el $85 \%$ de los encuestados estaban satisfechos o muy satisfechos con su formación doctoral. En tanto Parker-Jenkins (2018) en sus estudios ha determinado que uno de los elementos fundamentales en la concreción de estudios doctorales es la ayuda continua con la escritura académica por parte del profesorado, de igual modo señala que es fundamental establecer los alcances del programa desde sus inicios, dado que ello facilitaría a los estudiantes la finalización del programa que cursa. Otros estudios se han enfocado en la relación doctorando-tutor de tesis, un ejemplo de esto es el estudio de Hunter y Devine (2016) quienes, sugieren que la relación del doctorando con el supervisor de tesis es el factor más importante en lo que se refiere al agotamiento emocional de los estudiantes de doctorado, por lo que esta relación puede ser crucial en la finalización de la tesis doctoral. Pole et al., (1997) llegaron a la conclusión de que el supervisor de tesis puede ser el vínculo principal entre el individuo, el departamento y la institución, ya que la capacidad de este no solo proporcionaría al estudiante la información requerida, sino también entregaría la ruta por la cual el estudiante puede integrarse con la institución, lo que puede ayudar en el progreso del doctorando. Craft, Augustine-Shaw, Fairbanks y Adams-Wright (2016) relevan que las características de los tutores doctorales parecen influir, al menos en parte, en las actitudes generales de los estudiantes acerca de su experiencia de doctorado, lo que eventualmente podría afectar el progreso de un doctorando.

Los estudios internacionales develan el curso de la investigación en términos del nivel doctoral, no evidenciándose estudios que indaguen las expectativas de los doctorandos. Por tal motivo, esta investigación se propone indagar en aquellos elementos que relevan los futuros postgraduados a través de sus expectativas, con el fin de orientar la elaboración de los perfiles de egreso, para con ello mejorar la oferta académica chilena y su competitividad en una economía globalizada, ello porque "el desarrollo de la educación de nivel doctoral, es estratégico, pues constituye una fuente de bienestar tanto personal como colectivo, al generar importantes recursos en el mercado laboral para las personas y, al mismo tiempo, ser fuente de externalidades positivas al desarrollo del país" (Leiva, 2018, p. 40). En este sentido, el conocer las expectativas de los futuros doctores permitirá a las universidades ajustarse a nuevos desafíos y junto con ello elaborar programas capaces de desarrollar las habilidades tanto personales como sociales de sus futuros graduados, con el fin de gestar un postgraduado más acorde a las necesidades de un país en vías de desarrollo.

\section{METODOLOGÍA}

La metodología empleada. Esta ha sido dividida en tres subsecciones: objetivos, población y muestra, técnica de recolección y análisis de datos.

\section{Objetivos}

Para el desarrollo de este estudio, vinculado a las expectativas de los doctorandos de las áreas de educación y ciencias sociales chilenos, se proponen los siguientes objetivos de investigación (1) Identificar las expectativas de mayor frecuencia indicadas por los futuros doctores en educación y ciencias sociales chilenos sobre su formación en este nivel educativo. (2) Determinar y jerarquizar las razones de la elección de un programa de doctorado dictado en Chile. (3) Establecer las habilidades y proyecciones de los doctorandos en educación y ciencias sociales chilenos al graduarse como doctor/a. (4) Cuantificar la presencia y/o impacto de las variables acreditación y becas en la elección de un determinado programa doctoral. (5) Determinar según edad y nivel de doctorado (etapa de formación y etapa de tesis) las expectativas y proyecciones de los doctorandos. 


\section{Población y Muestra}

El muestreo es tipo aleatorio estratificado, cuyos estratos son caracterizados por los programas de doctorado en educación y por otro lado los doctorados en ciencias sociales que poseen como línea común investigaciones en educación. El tamaño de cada estrato está constituido respectivamente por 20 y 22 programas, identificándose en el caso de los programas de doctorado en educación 17 universidades y en ciencias sociales 23 . A partir de cada estrato y considerando el número total de estudiantes matriculados al año 2018, se determinó usando la fórmula de Namakforoosh (2000), que los respectivos tamaños muestrales con un error de estimación de un $5 \%$ y un nivel de confianza de un $95 \%$, siendo 44 y 50 estudiantes de doctorado. Es usada la formula antes indicada con el fin de minimizar el sesgo, preservando la aleatoriedad en el proceso. Es importante indicar que el número de estudiantes de los programas de doctorado que caracterizan la población de estudio es de 681 , por lo tanto, el tamaño muestral establecido supera el $10 \%$.

\section{Instrumento}

Para el levantamiento de los datos se confeccionó un instrumento de abstracción mixto, constituido por 10 preguntas de contexto y 10 preguntas específicas abiertas. Estas 10 últimas caracterizan tres dimensiones, una dimensión relativa a las expectativas de los doctorandos, otra a las razones de elección del programa que se cursa y la última a las proyecciones y elementos de contexto como becas y procesos de acreditación. Es de relevar, que con este instrumento son los doctorandos quienes levantan las alternativas de respuesta, las cual no han sido prefijadas como en test convencionales, minimizando aún más el sesgo. Las preguntas fueron elaboradas en función de los objetivos de investigación y de la literatura existente. Fue necesaria la elaboración de este instrumento, ya que no existe otro que aborde la temática. En términos de las características métricas del instrumento, esto es fiabilidad y validez, fueron determinadas usando las varianzas de las frecuencias de las 10 palabras más frecuentes, sin considerar conectores, a través de las aplicaciones wordcloud y ggplot del software R-project versión 3.5.1, obteniéndose como fiabilidad 0.71 y validez 0.70 (Gonzalez et al., 2016). Ambos resultados están por sobre el mínimo aceptado, por lo tanto, el instrumento tiene las características métricas deseadas. En la tabla 1 se presentan las preguntas que conforman el instrumento:

Tabla 1: Preguntas que componen el Instrumento de abstracción mixto.

\begin{tabular}{|c|c|}
\hline Preguntas de contexto & Preguntas específicas abiertas \\
\hline 1. ¿Cuál es su género? & $\begin{array}{l}\text { 1. ¿Cuáles son las características que debería tener un programa de } \\
\text { doctorado para cumplir con sus expectativas? }\end{array}$ \\
\hline 2. ¿Cuál es su edad? & $\begin{array}{l}\text { 2. Considerando la oferta de programas de doctorado tanto en Chile } \\
\text { como en el extranjero ¿Por qué eligió el programa que cursa? }\end{array}$ \\
\hline 3. ¿Cuál es su título profesional? & $\begin{array}{l}\text { 3. ¿Qué opinión le merecen los programas de doctorado dictados en } \\
\text { el extranjero en relación a los nacionales? }\end{array}$ \\
\hline $\begin{array}{l}\text { 4. Señale el tipo de Universidad de la cual } \\
\text { egresó en pregrado }\end{array}$ & $\begin{array}{l}\text { 4. ¿Cuál es su opinión acerca de las modalidades de los doctorados } \\
\text { dictados en Chile (a distancia, semipresencial y tiempo completo)? }\end{array}$ \\
\hline 5. ¿Tiene estudios de Magíster? & $\begin{array}{l}\text { 5. Considerando el número } 1 \text { como el más importante, enumere } \\
\text { aquellos elementos que para Ud. son los más relevantes dentro de un } \\
\text { doctorado para su formación como graduado. }\end{array}$ \\
\hline 6. Nombre del Doctorado que realiza & $\begin{array}{l}\text { 6. Al graduarse como Doctor ¿Cuáles son las habilidades que espera } \\
\text { haber desarrollado durante el proceso de formación doctoral? }\end{array}$ \\
\hline $\begin{array}{l}\text { 7. Universidad a la que pertenece el } \\
\text { Doctorado que realiza }\end{array}$ & 7. ¿A qué espera dedicarse al graduarse como Doctor? \\
\hline $\begin{array}{l}\text { 8. Señale el nivel de Doctorado en qué se } \\
\text { encuentra }\end{array}$ & $\begin{array}{l}\text { 8. ¿Qué opinión tiene sobre los procesos de aseguramiento de la } \\
\text { calidad -acreditación- a los cuales son sometidos los programas de } \\
\text { doctorado en Chile? }\end{array}$ \\
\hline $\begin{array}{lccc}\text { 9. ¿Posee publicaciones en revista } \\
\text { indexadas? }\end{array}$ & 9. ¿Qué rol tienen las "Becas" en la elección del doctorado que cursa? \\
\hline $\begin{array}{l}\text { 10. ¿Cuántas publicaciones indexadas } \\
\text { posee? }\end{array}$ & $\begin{array}{l}\text { 10. ¿Qué le incorporaría al doctorado que cursa para fortalecer su } \\
\text { formación? }\end{array}$ \\
\hline
\end{tabular}

Procedimiento de recogida y análisis de datos

La recolección de los datos se realizó de manera virtual, utilizándose la aplicación de Google denominada formularios. En ella se cargó el cuestionario, el que fue enviado vía correo electrónico a los coordinadores y/o directores de los programas pertenecientes a la muestra, con el fin de su reenvío a los estudiantes. De igual modo se envió directamente a lo doctorandos en aquellos casos donde se pudo acceder a sus emails. 
Junto con el cuestionario los doctorandos recibieron el protocolo de consentimiento informado, donde se explicitaron los objetivos de la investigación y la confidencialidad de los datos obtenidos. Para evitar la duplicación de respuestas, se utilizó como código identificador el correo electrónico de cada uno de los doctorandos constituyentes de la muestra, ya que no existen dos personas con la misma dirección e-mail. En términos, de los análisis generales se utilizó como técnica la cuantificación de la frecuencia de las 10 palabras más utilizadas en las respuestas abiertas de cada ítem del instrumento.

En lo referido a la comparación de los datos fue necesario definir un conjunto de indicadores, más allá de los convencionalmente existentes, en función de frecuencias, variabilidad y entropía: (1) Indicador de orden: dice relación a la cuantificación intervalar de la frecuencia media de las 10 palabras de mayor frecuencia, sin considerar conectores. (2) Indicador de variabilidad intervalar: dice relación con la variabilidad de las medias de las 30 palabras más frecuentes, considerando bloques de 10 palabras, es decir que tan diferente son las frecuencias luego de bloques de 10 palabras. (3) Indicador de Potencialidad: dice relación con la probabilidad de superar el $30 \%$ de las palabras utilizadas de mayor frecuencia, es decir, en función de la dinámica observada de las frecuencias de las palabras, establecer la probabilidad de superar el $30 \%$ de ellas, utilizando la distribución de conteo, Poisson. (4) Indicador de Entropía: dice relación con la cuantificación de la homogeneidad e incertidumbre en las frecuencias de las 10 palabras de mayor frecuencia. El software utilizado fue el R-project, en su versión 3.5.1, utilizándose las aplicaciones wordcloud y ggplot, el cual es de carácter libre.

\section{RESULTADOS}

Los resultados obtenidos de las respuestas abiertas del cuestionario fueron resumidos, a través, de gráficos de Wordle. Estos están presentados en función de los objetivos de investigación.

Objetivo 1: Identificar las expectativas de mayor frecuencia indicadas por los futuros doctores en educación y ciencias sociales chilenos sobre su formación en este nivel educativo.

En la figura 1 se grafican las expectativas de los doctorandos según el tipo de doctorado que realizan. En el caso de los estudiantes de doctorado del área de educación se evidencia que las expectativas están puestas tanto en el desarrollo de habilidades para la investigación como en el cuerpo académico del programa, colocándose de manifiesto la importancia de la adquisición de herramientas y habilidades para su formación doctoral. Se valora, además, la línea de investigación y el prestigio del programa que cursan. En el caso de las expectativas de los doctorandos en ciencias sociales el cuerpo académico ocupa la primera tendencia, seguido por las habilidades para la investigación. A diferencia de los doctorandos en educación, quienes valoran elementos como las revistas y publicaciones, el desarrollo de proyectos, la incorporación en equipos de trabajo, la internacionalización del programa y la existencia de recursos económicos y becas para la continuidad de este.
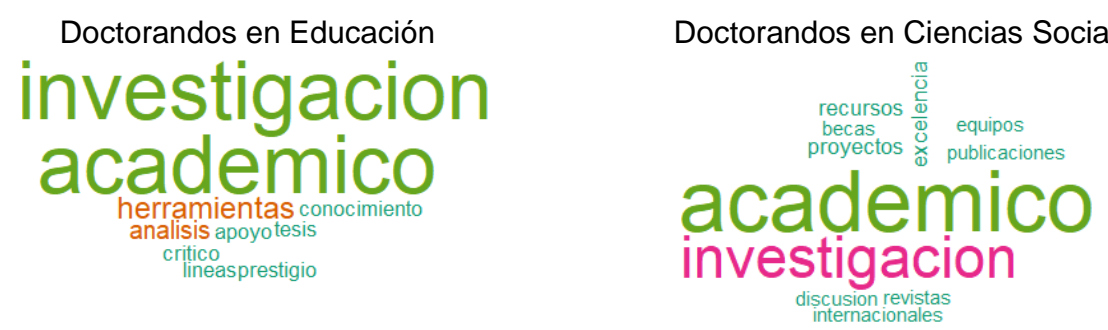

Fig. 1: Expectativas según el tipo de doctorado (educación y ciencias sociales)

Al realizar la comparación entre las expectativas de los doctorandos tanto en educación como en ciencias sociales, a partir de los indicadores objetivos definidos obtenemos los resultados presentados en la tabla 2:

Tabla 2: Comparación de las expectativas de los doctorandos en educación y ciencias sociales.

\begin{tabular}{|l|c|c|c|c|}
\hline $\begin{array}{l}\text { Tipo de } \\
\text { doctorado }\end{array}$ & Orden & $\begin{array}{c}\text { Indicador de } \\
\text { variabilidad }\end{array}$ & Potencialidad & Entropía \\
\hline Ciencias Sociales & {$[2.12 ; 11.47]$} & 0.75 & Prácticamente 0 & 2.96 \\
\hline Educación & {$[2.42 ; 10.14]$} & 0.72 & Prácticamente 0 & 3.29 \\
\hline
\end{tabular}

En lo expuesto en la tabla 2, y en términos del orden no se observan diferencias importantes, salvo una leve superioridad en la amplitud del intervalo para la media de las frecuencias de los doctorados en ciencias sociales. En lo referido al indicador de variabilidad se evidencia una diferencia 0.03 unidades, no registrando 
diferencias importantes entre los grupos. Por su parte, la entropía, en el caso de las expectativas de los doctorandos en ciencias sociales está direccionada a un elemento más específico - académicos del programa- en el caso de las expectativas de los doctorandos en educación existe un mayor número de palabras para referirse a estas. Respecto de la potencialidad, no existen diferencias y ambas afirman en términos probabilísticos que la dinámica de las frecuencias no visualiza nuevas palabras como para caracterizar los dos tipos de doctorados, dando así relevancia a las palabras de mayor frecuencia, dado que permiten dar cuenta de las expectativas de los doctores en formación.

Al comparar las expectativas de los estudiantes de ambas áreas del conocimiento, es posible apreciar la similitud en ambos grupos, relevándose la investigación y el cuerpo académico del programa. Como segundo grupo emerge el concepto de internacionalización, el que es apoyado por el de intercambio estudiantil y la discusión.

Objetivo 2: Determinar y jerarquizar las razones de la elección de un programa de doctorado dictado en Chile. Los doctorandos, tanto de educación como de ciencias sociales, relevan como elemento decidor en la elección del programa que cursan, la universidad que lo imparte, así como su dictación en territorio chileno, constituyéndose ambos elementos como los más importantes en la decisión que han tomado. En el segundo nivel jerárquico se encuentra la compatibilidad laboral con el programa y la línea de investigación en educación que posee este. En tercer lugar, se menciona la importancia de la investigación, para finalmente señalar la flexibilidad, el cuerpo académico y la cercanía geográfica. Esta jerarquización se grafica en la figura 2.

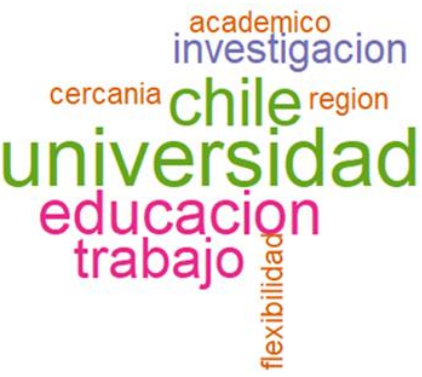

Fig. 2: Razones para la elección de un programa de doctorado en Chile.

Objetivo 3: Establecer las habilidades y proyecciones de los doctorandos en educación y ciencias sociales chilenos al graduarse como doctor/a. En términos de los objetivos que esperan alcanzar los doctorandos al concluir su formación, estos concuerdan en que lo más relevante es la investigación como competencia, siguiéndole en importancia el logro de habilidades de análisis, escritura académica, conocimiento científico y autonomía. Las frecuencias mencionadas están presentadas en la figura 3:

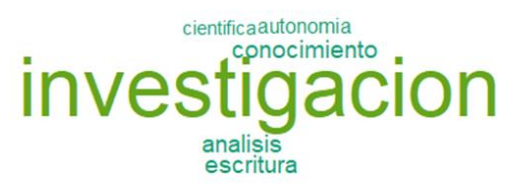

Fig. 3: Objetivos que esperan alcanzar los doctorandos al finalizar su formación.

En lo referido a las proyecciones laborales de los doctorandos al concluir el programa que cursan, estos señalan en primer lugar la investigación como actividad a desarrollar y en segundo, el trabajo académico en entidades de educación superior.

Objetivo 4: Cuantificar la presencia y/o impacto de las variables acreditación y becas en la elección de un determinado programa doctoral. En la figura 4, se evidencia la opinión de los doctorandos sobre la acreditación de los programas de doctorado. En este refieren que la acreditación es un proceso que vela por la calidad de un programa, a través, de estándares lo que es considerado como importante y necesario para este nivel educativo. De igual modo, se grafica el elemento becas, las que se presentan como fundamentales para el desarrollo de estudios de nivel doctoral, siendo relevante optar a ellas para financiar estos estudios. 


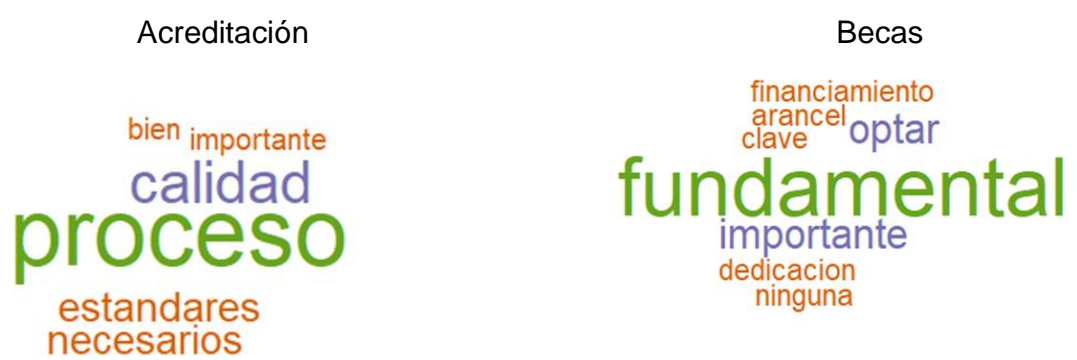

Fig. 4: Variables de acreditación y becas.

Objetivo 5: Determinar según edad y nivel de doctorado (etapa de formación y etapa de tesis) las expectativas y proyecciones de los doctorandos. Expectativas: En la figura 5, se grafican las expectativas de los doctorandos según dos grupos etarios, a saber, menores o iguales a 35 años y mayores de 35 . En el primer grupo, las expectativas sobre un programa doctoral están puestas en el cuerpo académico, siguiendo la investigación y en tercer lugar temas asociados al financiamiento. En el caso de los mayores de 35 años la importancia recae tanto en el cuerpo académico como en la investigación, se relevan en un segundo grupo las habilidades, la participación en proyectos y la tesis doctoral.

En relación a los indicadores cuantitativos para la comparación de los grupos antes indicados, se presenta la Tabla 3.
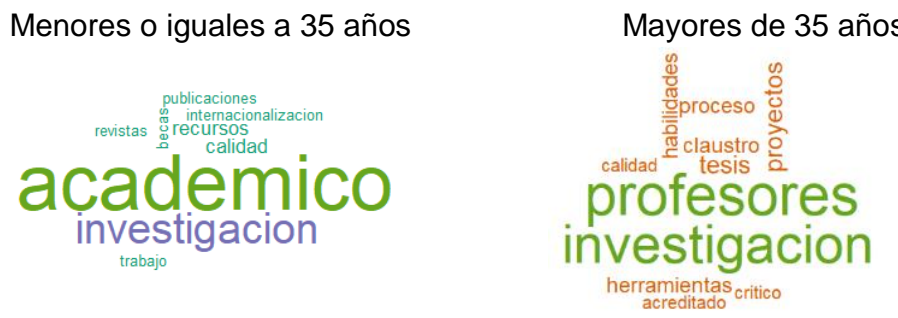

Fig. 5: Expectativas según edad.

Tabla 3: Comparación de las expectativas de los doctorandos según edad.

\begin{tabular}{|l|c|c|c|c|}
\hline Edad & Orden & Variabilidad & Potencialidad & Entropía \\
\hline Menores o iguales 35 años & {$[1.6 ; 13.4]$} & 0.83 & Prácticamente 0 & 2.3 \\
\hline Mayores 35 años & {$[3.4 ; 8.4]$} & 0.66 & Prácticamente 0 & 3.97 \\
\hline
\end{tabular}

En términos de orden, en la tabla 3, se observa una notación intervalar de menor amplitud en relación a la puntuación media de las frecuencias para el caso de los mayores de 35 años, siendo consistente con el indicador de variabilidad, evidenciado una diferencia de 0.17 unidades, es decir, se cuantifica mayor claridad en las afirmaciones referidas por los mayores de 35 años. Por otro lado, la potencialidad es prácticamente nula en ambos casos, lo que evidencia en términos de probabilidades que no se incrementarán las frecuencias de las palabras. Finalmente, para la entropía, se visualiza una superioridad en los mayores de 35 años, reflejando una mayor cantidad de expectativas de alta frecuencia y en el caso de las expectativas de los doctorandos menores o iguales a 35 años está direccionada a un elemento más específico - académicos del programa. En la figura 6, se grafican las expectativas de los doctorandos según la etapa doctoral en la que se encuentran: etapa de formación y etapa de tesis. En el primer grupo las expectativas están puestas sobre los profesores que imparten clases en esta etapa, siguiendo la investigación y en tercer lugar la formación propiamente tal. En el caso de los doctorandos en etapa de tesis, los profesores siguen ocupando la primera referencia, aunque aumenta la importancia de la investigación.
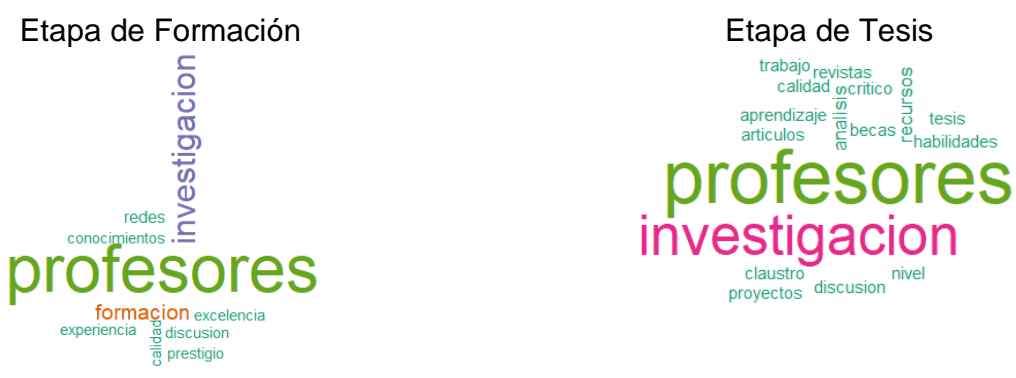

Fig. 6: Expectativas según etapa doctoral. 
En la tabla 4, en función del orden no existen diferencias a destacar, lo mismo que en el indicador de variabilidad cuya diferencia es de 0.07 unidades. En términos de potencialidad, los estudiantes en etapa de formación tienen una probabilidad de modificación o de nuevas expectativas nula y en el grupo de los tesistas está modificación es prácticamente nula, siendo esta cuantificación más tajante en el caso de estos últimos. Por su parte, la entropía en el caso de las expectativas de los doctorandos tesistas está direccionada a un elemento más específico en comparación a los estudiantes en formación.

Tabla 4: Comparación de las expectativas de los doctorandos etapa doctoral.

\begin{tabular}{|l|c|c|c|c|}
\hline \multicolumn{1}{|c|}{ Etapa } & Orden & Indicador de variabilidad & Potencialidad & Entropía \\
\hline Formación & {$[2 ; 11.02]$} & 0.74 & Prácticamente 0 & 3.14 \\
\hline Tesis & {$[2.1 ; 11.32]$} & 0.67 & 0 & 2.91 \\
\hline
\end{tabular}

Habilidades: En la figura 7, se grafican las habilidades que los doctorandos esperan desarrollar según su edad: menores o iguales a 35 años y mayores de 35 . En ambos grupos las habilidades vinculadas con la investigación son las más importantes, relevándose en el segundo grupo la adquisición de conocimientos.
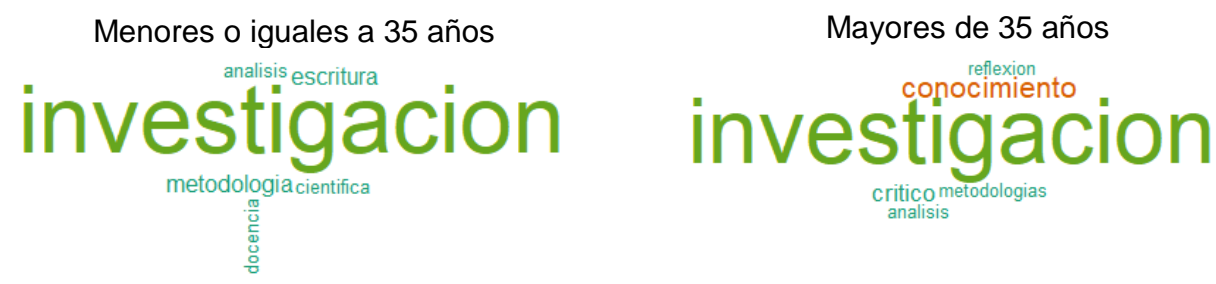

Fig. 7: Habilidades a desarrollar según edad.

Tabla 5: Comparación de las habilidades a desarrollar según edad.

\begin{tabular}{|l|c|c|c|c|}
\hline \multicolumn{1}{|c|}{ Edad } & Orden & Indicador de variabilidad & Potencialidad & Entropía \\
\hline Menores o iguales 35 años & {$[0.23 ; 10.56]$} & 0.67 & 0.18 & 3.77 \\
\hline Mayores 35 años & {$[0.55 ; 11.24]$} & 0.75 & 0.19 & 3.12 \\
\hline
\end{tabular}

En la tabla 5, en términos del orden prácticamente la amplitud intervalar se mantiene, salvo un pequeño desfase, un aumento en el caso de los mayores de 35 años. En lo referido al indicador de variabilidad se evidencia una diferencia 0.12 unidades, no registrando diferencias importantes entre los grupos. En relación a la potencialidad, en ambos grupos esta es prácticamente idéntica, vale decir, las probabilidades de incrementar las habilidades a desarrollar están bajo el $20 \%$. Por su parte, la entropía en el caso de ambos grupos posee una tendencia hacia la heterogeneidad, es decir a ampliar el abanico de habilidades. En lo referido a las habilidades, ambos grupos señalan que las habilidades vinculadas con la investigación son las más importantes en su formación doctoral.

En la tabla 6, y en lo referido al orden, la amplitud del grupo en formación es menor por tanto la media de las frecuencias presenta menor variabilidad. En relación al indicador de variabilidad se evidencia una diferencia 0.17 unidades, no registrando diferencias importantes entre los grupos. En términos de potencialidad, en los estudiantes en etapa de formación la probabilidad de modificación es baja, en cambio en el grupo de los estudiantes tesistas la probabilidad de modificación y/o emergencia de nuevas habilidades es más alta. Por su parte, la entropía, en ambos grupos tiende a un conjunto de palabras más amplias, siendo superior en el caso de los doctorandos en formación.

Tabla 6: Comparación de las habilidades a desarrollar según etapa de formación doctoral.

\begin{tabular}{|l|c|c|c|c|}
\hline \multicolumn{1}{|c|}{ Etapa } & Orden & Indicador de variabilidad & Potencialidad & Entropía \\
\hline Formación & {$[1.18 ; 9.81]$} & 0.67 & 0.15 & 3.82 \\
\hline Tesis & {$[0.01 ; 12.58]$} & 0.84 & 0.51 & 3.30 \\
\hline
\end{tabular}

Proyecciones: En la figura 8, se grafican las proyecciones de los doctorandos al obtener el grado académico. Para la comparación se consideran los grupos etarios antes mencionados. En el grupo de menores o iguales a 35 años, la investigación y la docencia como actividad a desempeñar se posicionan de igual modo. En el segundo grupo la docencia pierde relevancia aumentando así la investigación, constituyéndose como la actividad principal a desarrollar. 
Fig. 8: Proyecciones según edad.

En términos del orden, en la tabla 7, la amplitud intervalar es menor para los doctorandos menores o iguales a 35 años, lo que evidencia representatividad de la frecuencia media. En lo referido al indicador de variabilidad se evidencia una diferencia 0.02 unidades, no registrando diferencias importantes entre los grupos. En relación a la potencialidad, ambos grupos evidencian una alta probabilidad de cambiar sus proyecciones. Por su parte, la entropía en el caso de ambos grupos existe una tendencia hacia la investigación y a la docencia, siendo más clara en el caso de los mayores de 35 años.

Tabla 7: Comparación de las proyecciones según edad.

\begin{tabular}{|l|c|c|c|c|}
\hline \multicolumn{1}{|c|}{ Edad } & Orden & Indicador de variabilidad & Potencialidad & Entropía \\
\hline Menores o iguales 35 años & {$[0.29 ; 10.5]$} & 1.02 & 0.99 & 1.88 \\
\hline Mayores 35 años & {$[0.25 ; 14.34]$} & 1.00 & 0.99 & 1.02 \\
\hline
\end{tabular}

Las proyecciones de los doctorandos tras al obtener el grado académico, según la etapa de formación doctoral en la que se encuentran, se expresa del siguiente modo: en el grupo de estudiantes en formación, la actividad a desarrollar principalmente es la de académico en una institución universitaria y como segunda la investigación. Es de relevar que ambas actividades se desean llevar a cabo en una institución de carácter pública. En el grupo de estudiantes tesistas la investigación adquiere relevancia por sobre la docencia, apareciendo un tercer grupo que opta por la gestión.

Tabla 8: Comparación de las proyecciones según etapa de formación doctoral.

\begin{tabular}{|l|l|c|c|c|}
\hline \multicolumn{1}{|c|}{ Etapa } & Orden & Variabilidad & Potencialidad & Entropía \\
\hline Formación & {$[0.59 ; 13.20]$} & 1.10 & 0.99 & 1.41 \\
\hline Tesis & {$[0.29 ; 14.10]$} & 1.16 & 0.99 & 1.21 \\
\hline
\end{tabular}

En la tabla 8 y en lo referido al orden las amplitudes prácticamente no difieren, sin embargo, es superior en el caso de los alumnos en etapa de tesis. En términos de variabilidad se evidencia una diferencia 0.06 unidades, no registrando diferencias importantes entre los grupos. En relación a la potencialidad, ambos grupos evidencian una alta probabilidad de cambiar sus proyecciones. Por su parte, la entropía en el caso de ambos grupos tiene una tendencia hacia la investigación y a la docencia, siendo más clara en el caso de los doctorandos tesistas.

\section{DISCUSIÓN}

En Chile, los programas de doctorado tanto de educación como de ciencias sociales experimentaron un desarrollo tardío en comparación a otras áreas disciplinarias. Ello obedece en gran medida a dos factores. El primero dice relación con el inicio de la formación doctoral en territorio chileno, el cual sólo data de 1947 y el segundo, vinculado con la necesidad de consolidar las áreas tradicionales del conocimiento para luego generar programas en áreas menos desarrolladas como lo fueron educación y ciencias sociales (Baeza, 2017). En la actualidad el número de programas doctorales dictados en ambas áreas asciende a 70 y se encuentran repartidos en diferentes regiones del país (Ministerio de Educación, 2018). El surgimiento de diferentes programas doctorales, en gran medida obedece a la necesidad de dotar al país de avances científicos y tecnológicos (Meissner, Gokhberg y Shmatko, 2016)

La formación de futuros doctores representa una oportunidad para el desarrollo país, pues como lo menciona Jiménez- Ramírez (2017) estos son los recursos humanos llamados a generar investigación científica y original, y son las personas que han de liderar, a través de sus conocimientos los diferentes procesos a los que se enfrenta la sociedad en un contexto globalizado. Las expectativas de los doctorandos, permiten no sólo conocer lo que se espera de la formación doctoral, sino que también otorga la posibilidad a los programas doctorales de evaluar el tipo de formación que se ofrece, vale decir, modificar o incorporar elementos que harán del programa más competitivo y además permitirá realizar intervenciones en pos de la mejora (Culver, 2018). En términos de expectativas los doctorandos tanto de educación como de ciencias sociales señalan como fundamentales el desarrollo de habilidades para la investigación. El desarrollo de estas habilidades resulta crucial para la escritura académica y la futura publicación de investigaciones originales (Paris, Wasik y Turner, 2016). Estudios internacionales como el de Parker-Jenkins (2018) señalan que uno de los elementos fundamentales para una finalización exitosa de estudios doctorales es el apoyo continuo por parte de los programas de doctorado en lo que se refiere a la escritura académica. 
Otro elemento relevado por los doctorandos es el cuerpo académico del programa, pues se considera a este de gran importancia para la formación doctoral, ya que se les eleva a la categoría de referentes teóricos e investigativos. En este sentido, Munthe y Magne (2015) señalan que el éxito de los trabajos de tesis se sustenta en gran medida por la presencia de profesores-investigadores calificados y activos. Thompson, Ballenger y Templeton (2018) han concluido en sus estudios que la relación tutor- estudiante es un factor fundamental en el rendimiento de los doctorandos en un programa. En el estudio desarrollado por Hunter y Devine (2016) se sugiere que la relación del doctorando con el supervisor de tesis es el factor más importante en la finalización de la tesis doctoral, ya que, este proceso conlleva un gran agotamiento emocional, el cual puede ser mitigado por la relación que se genera con el tutor y en cierta medida evitaría la deserción de los doctorandos. Estos antecedentes explican en gran medida porque los procesos de acreditación chilenos evalúan la conformación de los claustros académicos de los programas doctorales, dado que se espera asegurar su idoneidad para la formación en este nivel educativo. Los doctorandos además esperan en su ciclo de formación adquirir herramientas y habilidades que les permitan desarrollarse posteriormente. Resulta relevante mencionar que los doctorandos en ciencias sociales valoran elementos como las revistas y publicaciones, lo que está vinculado directamente con el desarrollo de la investigación, así también la ejecución de proyectos, los cuales representan en Chile una oportunidad de conseguir financiamiento para el trabajo investigativo, de igual modo consideran la incorporación en equipos de trabajo, la internacionalización del programa y el financiamiento de los estudios doctorales, a través de becas, lo cual adhiere a los señalado por estudiantes en otras latitudes, resultados que son presentados en los estudios de Shapiro, Hudson y Downey (2017).

En lo referido a las razones por las cuales se eligió un programa de doctorado dictado en Chile, considerando la gran oferta académica que existe a nivel mundial, los doctorandos señalaron que el elemento más importante es la universidad que lo imparte y el segundo es que la universidad esté en territorio chileno. Ambos dan cuenta del interés de estos futuros doctores de formarse en territorio nacional, lo que se explicaría por las razones señaladas en segundo orden jerárquico, a saber, la compatibilidad del programa elegido con las actividades laborales, lo que coloca en evidencia que los doctorandos no sólo se dedican a sus estudios, sino que además poseen un trabajo remunerado. A lo que se agrega la flexibilidad horaria del doctorado elegido. Todos estos elementos nos permiten establecer que los doctorandos encuestados no poseen una dedicación exclusiva al programa que cursan, lo que en gran medida se relaciona con los sistemas de financiamiento que existen en Chile para cursar estudios de postgrados, donde la acreditación es requisito fundamental para acceder a una Beca doctoral.

Mohammad (2017) señala que la existencia de becas podría asegurar la finalización de un programa doctoral, dado que la dedicación a labores profesionales resta tiempo para la conclusión de una tesis de nivel doctoral. Es importante recordar, que Becas Chile solo están abiertas para estudiantes que estén matriculados en un programa doctoral acreditado, excluyendo a un número no menor de estudiantes que se encuentran en programas no acreditados. Es de relevar que los doctorandos no señalaron la acreditación del programa como un elemento a considerar en la elección de este, aunque este proceso es visto como un regulador de la calidad del programa, a través, de una serie de estándares establecidos, lo que resulta importante y necesario para este nivel educativo. La acreditación y las becas, como ya se ha mencionado, tienen una estrecha vinculación, dado que la entrega de financiamiento por parte del Estado se da en función de la acreditación de un programa. Si bien es cierto, los programas poseen becas internas a las cuales es factible postular estando matriculado en él, no existiendo sistemas alternativos para los programas no acreditados. En términos de becas, los doctorandos señalan a estas como fundamentales para el desarrollo de estudios de nivel doctoral, siendo relevante optar a ellas para financiar estos estudios. Se evidencia tensión en las observaciones que realizan los doctorandos sobres acreditación y becas, por lo que resultaría interesante indagar por qué la acreditación no es un factor determinante en la elección de un programa, si de ella dependen los recursos económicos.

Los doctorandos no solo esperan desarrollar una serie de habilidades al finalizar el programa doctoral, sino que también poseen proyecciones sobre su futuro laboral. En términos de habilidades se espera alcanzar habilidades de investigación, de análisis, de escritura académica y autonomía. Resulta interesante que los doctorandos mayores de 35 años valoren además de las competencias investigativas y las asociadas a estas, el conocimiento como parte fundamental de su proceso de formación, lo que ocurre de igual modo con los estudiantes que se encuentran en la etapa de formación, lo que es coherente con el proceso educativo que viven. Los estudiantes en etapa de tesis otorgan mayor importancia a la investigación y a sus habilidades, puesto que se encuentran en este proceso. En propuestas internacionales como las de Nebot (2009) el graduarse como doctor significa necesariamente realizar investigación original para contribuir a una determinada área del conocimiento, siendo la competencia fundamental en un programa doctoral.

Finalmente, en lo relacionado con las proyecciones, los doctorandos manifiestan que la investigación y la docencia son las tareas que han de realizar cuando obtengan el grado académico. No obstante, resulta 
interesante observar como una adquiere mayor relevancia que la otra según la edad y la etapa doctoral en la que se encuentran. Por ejemplo, en el grupo de estudiantes menores o iguales a 35 años, la investigación y la docencia se posicionan de igual modo, no así en el grupo de estudiantes mayores de 35 años, donde la docencia pierde relevancia aumentando el interés por la investigación. En el caso de los estudiantes que se encuentran en etapa de formación la actividad a desarrollar principalmente es la de académico, referenciándose como segunda la de investigación, en ambas actividades los doctorandos señalan que estas se desean llevar a cabo en una institución de carácter público. En el grupo de estudiantes tesistas la investigación adquiere relevancia por sobre la docencia, apareciendo un tercer grupo que opta por la gestión. Los tesistas muestran una mayor tendencia a la investigación, lo que se podría explicar porque se encuentran en pleno trabajo investigativo. Las expectativas de los doctorandos se alinean con los planteamientos actuales, donde los doctorados comienzan a adquirir una "orientación formativa más centrada en dotar de competencias para la investigación que incidir exclusivamente en la formación del profesorado universitario" (Jiménez- Ramírez, 2017, p.408)

\section{CONCLUSIONES}

De los resultados presentados, sus análisis y posterior discusión se obtienen las siguientes conclusiones con respecto a las expectativas de los doctorandos sobre su formación doctoral: (1) las expectativas manifestadas por los encuestados son coherentes con las demandas actuales para este grado académico, lo que se ha expresado en documentos emanados desde el proceso de Bolonia y por los diferentes autores citados en este estudio (2) el desarrollo de habilidades para la investigación son consideradas por los doctorandos como cruciales para su formación y posterior desarrollo profesional, ello nos lleva a concluir que deben ser consideradas en la etapa de formación de los postgraduados (3) el éxito de un programa doctoral se encuentra vinculado entre otros factores a los académicos que componen el claustro, dado que son estos quienes inciden en el éxito de las tesis doctorales y en el rendimiento de los doctorandos, lo que ha sido estudiado ampliamente en países anglosajones, lo cual representa un referente para el medio chileno (4) las becas son fundamentales para la concreción de los estudios doctorales y para el desarrollo de la investigación, ya que permiten al doctorando su dedicación exclusiva a esta actividad (5) la acreditación de un programa doctoral no es un factor clave en la elección de este por parte de un aspirante a doctor, no así, el prestigio de la universidad.

\section{REFERENCIAS}

Baeza, P., Diversidad y diferenciación en la oferta de programas de doctorado en Chile, Calidad en la educación, 47, 179214 (2017)

Cancino, V. y R. Schmal, Sistema de Acreditación Universitaria en Chile: ¿Cuánto hemos avanzado?, Estudios Pedagógicos, 40(1), 41-60 (2014)

Chiappa, R. y A. L. Muñoz, Equidad y capital humano avanzado: Análisis sobre las políticas de formación de doctorado en Chile, Psicoperspectivas, 14(3), 17-30 (2015)

Comisión Nacional de Acreditación. Guía para la elaboración del informe de autoevaluación de programas de Doctorado, Comisión Nacional de Acreditación, Chile (2013)

Corvalán, J., A. Falabella y M.T. Rojas, El doctorado en educación: un ejemplo de desregulación en el campo de la educación superior en Chile, Calidad en la Educación, (34), 15-42 (2011)

Craft, C.M., D. Augustine-Shaw, A. Fairbanks y G. Adams-Wright, Advising doctoral students in education programs. NACADA Journal, 36(1), 54-65 (2016).

Culver, J., Pursuing the Professoriate: The Academic Career Development of Black Female Doctoral Students at Predominantly White Institutions, Dissertations, 757 (2018)

González, J., F. Viveros y C. Carvajal, Coeficientes edumétricos para la validez y dificultad de un test: Propuesta, Estudios Pedagógicos, 42(3), 448-467 (2016)

Horgué, C., La ordenación del doctorado, Revista de Administración Pública, 189, 365-401 (2012)

Hunter, K.H. y K. Devine, Doctoral students' emotional exhaustion and intentions to leave academia, International Journal of Doctoral Studies, 11(2), 35-61 (2016)

Jiménez -Ramírez, M., Convergencia europea e internacionalización del Doctorado: panorámica sobre las tendencias de cambio en España, Estudios Pedagógicos, 43(1), 405-422 (2017)

Krauskopf, M., Indicadores cuantitativos de los doctorados conferidos en el país. ¿Falta de atención o expresión de subdesarrollo? Calidad en la Educación, (18), 47-59 (2003)

Leiva, A., Ejes para la elaboración de una política para la educación superior, Calidad en la Educación, (22), 39-52 (2018)

Manzo, L., M. Rivera y A. Rodríguez, La educación de posgrado y su repercusión en la formación del profesional iberoamericano, Educación Médica Superior, 20(3) (2006) 
Meissner, D., L. Gokhberg y N. Shmatko, The Meaning of Doctorate Holders for Human Capital Development of Nations, The Science and Technology Labor Force, Springer International Publishing, 343-350, Cham, Alemania (2016)

Ministerio de Educación, ¿Hacia dónde avanza el sistema educativo en Chile? Análisis de las recomendaciones OCDE contenidas en Evaluaciones de Políticas Nacionales de Educación: Educación en Chile (2004-2016) en el contexto de la Reforma en marcha, Ministerio de Educación, Santiago, Chile (2017)

Ministerio de Educación, Base de datos Matrícula Histórica- Informe SIIES, Ministerio de Educación, Chile (2018)

Mohammad, I., Importance of Supervisory in Educational Progress of PhD Students, Journal of Humanities Insight 1.2, 7688 (2017).

Möller, I. y H. Gómez, Coherencia entre perfiles de egreso e instrumentos de evaluación en carreras de educación básica en Chile, Calidad en la educación, (41), 17-49 (2014)

Munthe, E. y M. Magne, Research based teacher education, Teaching and teacher education, 46, 17-24 (2015)

Namakforoosh, M., Metodología de Investigación, 203-303, Prentice-Hall Hispanoamericana, Ciudad de México, México (2000)

Organización para la Cooperación y el Desarrollo Económico Panorama de la Educación. Indicadores de la OCDE 2017, OCDE, Madrid, España (2017)

Paris, S., B. Wasik y J. Turner, The Development of Strategic Readers, Handbook of Reading Research Routledge, Londres, Reino Unido (2016)

Parker-Jenkins, M., Mind the gap: developing the roles, expectations and boundaries in the doctoral supervisor-supervisee relationship, Studies in Higher Education, 43(1), 57-71 (2018)

Pole, C. J., A. Sprokkereef, R.G. Burgess y E. Lakin, Supervision of doctoral students in the natural sciences: Expectations y experiences, Assessment y Evaluation in Higher Education, 22(1), 49-63 (1997)

Sánchez-Medina, A., C. García-Ventura y E. Ayuga-Tellez, Análisis y Comparación de la Información mostrada en las Webs Españolas y Extranjeras Relativa a Programas de Doctorado en el Área Agroforestal, Formación Universitaria, 9(5), 97-106 (2016)

Shapiro, V. B., K.D. Hudson y M.M. Downey, Institutional Expectations, Opportunities, y Interest in the Professoriate: $A$ Mixed-Methods Examination of Satisfaction Among Doctoral Students in Social Work, Journal of Social Work Education, 53(3), 520-534. (2017)

Thompson, J., J. Ballenger y N. Templeton, Examining Quality Elements in a High Education Fully Online Doctoral Program: Doctoral Students' Perceptions, International Journal of Educational Leadership Preparation, 51, 51-63 (2018)

Van der Weijden, I., C. Teelken., M. de Boer y M. Drost, Career satisfaction of postdoctoral researchers in relation to their expectations for the future, The International Journal of Higher Education Research, 72(1), 25-40 (2016)

Vera, P., Historia y estado actual de la formación doctoral en Chile, Revista Digital Universitaria, 11(5), 3-17 (2010) 